\title{
Psychological attentional characteristics based on TEST2DRIVE test battery and age as a factor of drivers distraction in LCT and 3VPT simulator scenarios
}

\author{
Adam Tarnowski ${ }^{1, *}$, and Krzysztof Szostok ${ }^{2}$ \\ ${ }^{1}$ Institute of Motor Transportation, Jagiellońska 80, 03-301 Warsaw, Poland \\ ${ }^{2}$ ALTA Ltd., Olimpijska 7, 41-100 Siemianowice Śląskie
}

\begin{abstract}
Drivers distraction analysis should take into account capabilities of road users with the weakest performance. The first issue was to determine by Test2Drive psychological testing system, empirical criteria of limited attention performance. The second issue was the level of influence of distracting roadside advertisement on driving performance in standard simulator tasks - three vehicle platoon task and lane changing task. The attentional performance has been established by PUT test. This method, based on conjunctive search task was an indicator of speed and accuracy of visual inspection. In the experiment distraction mode has been established at three levels: on roadside billboards red dot has been absent or exposed for 1 or 2 second. In first analysis a group of about 17000 professional drivers, aged 18-84 form Test2Drive database were selected. In a simulator experiment a group consisted of 30 drivers in age 20-60. As expected, attention performance decreased with age. Markers of impaired attentional performance have been established. In the experiment, no significant effects have been found in 3VPT paradigm. In LCT paradigm, a significant interaction of attention and distraction mode has been observed - drivers with symptoms of attentional malfunctions presented significant problems with maneuver accuracy. The experiment allowed to prove that attentional performance led to higher distraction when stimuli analogical to roadside advertisement were presented. The observed effect was statistically significant but not so strong as in had been expected, what can be explained by a small sample size, in which attention disorders rate were not high. Even with its limitations the experiment proves a simple thesis - roads users with limited attention performance are safer when no salient distractors are disturbing them.
\end{abstract}

\section{Introduction}

The drivers distraction is commonly defined as "occurring when a driver's attention is, voluntarily or involuntarily, diverted away from the driving task by an event or object to the

\footnotetext{
*Corresponding author: adam.tarnowski@ipsyt.pl
} 
extent that the driver is no longer able to perform the driving task adequately or safely" [1] (p. 380). This general definition include physical (secondary moto tasks), auditory, visual and cognitive distraction. Roadside advertisement is often treated as an important distraction source [2]. Individual resistance to distraction depends on several factors, like cognitive resources and attention system characteristics. We have to assume, that many road users are sensitive to distraction and they should have an opportunity to drive safely. The environment itself should be safe even for people with low performance, as long they can safely drive.

The attention deficits are commonly perceived as a problem of elderly drivers [3]. Problem is not as simple as it may appear based on common observations and simplified analysis. Madden's [4] research show that the top-down search is relatively constant, while only bottom-up functions are deteriorating. Kolodziejczyk [5] in his works demonstrates how weakening of attention and fluid intelligence can be compensated with increasing experience. This ability is progressing even up to the age of 70. Older drivers are therefore still fit to drive, but, taking into account weak bottom-up attentional performance, they can be much more vulnerable for intruding salient roadside advertisement.

The first observations concerning roadside advertisement influence on drivers distractions have been made in 1950 by McMonagle [6]. In his study, Author analyzed a 112 $\mathrm{km}$ long road in Michigan, divided into short sections. Next, during a period of three years, all accidents and incidents were registered (basing on police reports). In effect, a correlation between accident rate and road environment complexity has been found. The most frequent accidents happened on junctions with large number of billboards.

Recently, the problem has been investigated by Bendak and Al-Saleh [7] in their experiment a two $9 \mathrm{~km}$ routes have been implemented in a simulator. The two sections varied with density of roadside advertisement. All indicators of distracted driving (lane deviation, unsafe crossing of intersections, exceeding speed, time-to-collision with preceding vehicle and turn signal usage) were higher on a road with billboards, and two first differences were significant. $50 \%$ of subjects in debriefing confirmed fact of distraction, and $22 \%$ assessed the road situations as dangerous. In a Polish large sample CAWI pool study [8] $42,8 \%$ of responders confirmed the fact of being distracted by roadside advertisements. $37 \%$ of them were rather often, very often or always distracted, and 29,7\% experienced diminished situational awareness. The more representative review of research, including pool data, simulator experiments, laboratory experiments and real road research can be find in a work of Wallace [9].

Reported study is a part of the large project, exploring impact of individual differences and external conditions like time of exposition, luminance, content and location of roadside advertisement on drivers distraction. In the current article the relationship between attention performance and distracted driving has been explored. I the first part indicator and cut point for attention performance is established. In the next part of the study the moderating effect of attention on relationship between distractor exposition time and driving performance has been investigated.

\section{Material and methods}

First part of the study has been devoted to evaluation of PUT attention test, and establishing a cut point for poor attentional performance. The PUT test, a part of Test2Drive ${ }^{\mathrm{TM}}$ traffic psychology battery, is founded on Treismann concept of conjunctive search [10]. The basic assumption of the theory is a necessity of serial, working memory based processing of the search task requiring finding an object defined by two features. In singleton search, subject can scan the visual field parallelly, using peripheral or parafoveal processing - but in conjunctive search one can recognize an object only with central perception, what forces saccadic movements across visual field and serial comparing object with mental 
representation. The test material requires pointing a "vertical black triangle" among the set of distractors. Matrices contain 4, 9, 16 or 25 stimuli. In a half of them, the target is missing, and then correct answer is to press a "absent" button. Basic test variables are mean time of inspection for tables without target and overall percentage of correct answers. The test is presented on 23 " touch screen.

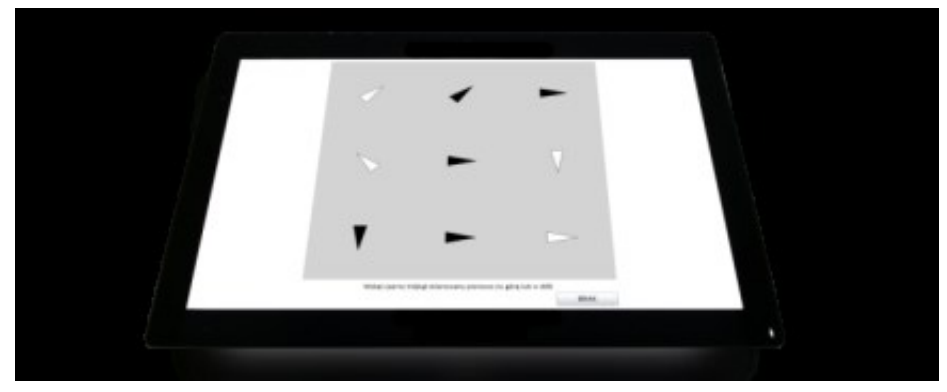

Fig. 1. Sample PUT task. Subject is asked to point a vertical black triangle with a finger on touch screen.

The PUT test [11] has been introduced to traffic psychology in nearly 100 laboratories. The database contains about 20000 datasets and is still increasing. Additionally, the drivers assessed as unfit to drive can be selected out from the set. In result, 17652 active drivers aged $18-85$ years, $10.5 \%$ women, have been included in analyses.

In the second part of the study, established cut point has been used in simulator experiment. 60 drivers, 30 men and 30 women, representing in equal proportions age groups under 25 (unexperienced), 30-40 years of age (optimal) and 55-65 senior drivers. The experimental task based on 3 Vehicle Platoon Task (3VPT) and Lane Change Task (LCT) [11-13]. In LCT subject is required to change lane as soon as possible when a sign is displayed on a sideway, and in $3 \mathrm{VPT}$ subject is required to keep constant speed and equal distance between proceeding and following car.

In $3 \mathrm{VPT}$ experimental paradigm the accuracy of driving was measured with following variables:

- $\quad$ Mean speed

- $\quad$ Standard deviation of speed (as an indicator of speed instability)

- Mean time to collision

- Standard deviation of time to collision (as an indicator of car position in a column instability)

- Mean distance to lane centerline (as an indicator of road position)

- Standard deviation of distance to lane centerline (as an indicator of road position instability).

In LCT paradigm the driving performance has been assessed with use of following variables:

- Ideal range: an area between ideal trace for the maneuver and a real one;

- Reference range: an area between optimal trace of maneuver, adjusted for initial position on the road, and a real one.

- Reaction time (measured between lane change sign exposition and first reaction.

- $\quad$ Speed variability (Standard deviation of speed registrations)

Three experimental conditions have been introduced. In each of them, the roadside billboards have been positioned. Subjects were divided randomly into three independent groups, with assigned experimental conditions:

- In every billboard a red dot has been displayed for 1 second

- In every billboard a red dot has been displayed for 2 seconds

- In control group, no dots were displayed. 


\section{Results}

In part one, the test variables have been analyzed by decades of age. Graph below and ANOVA table presents the results:

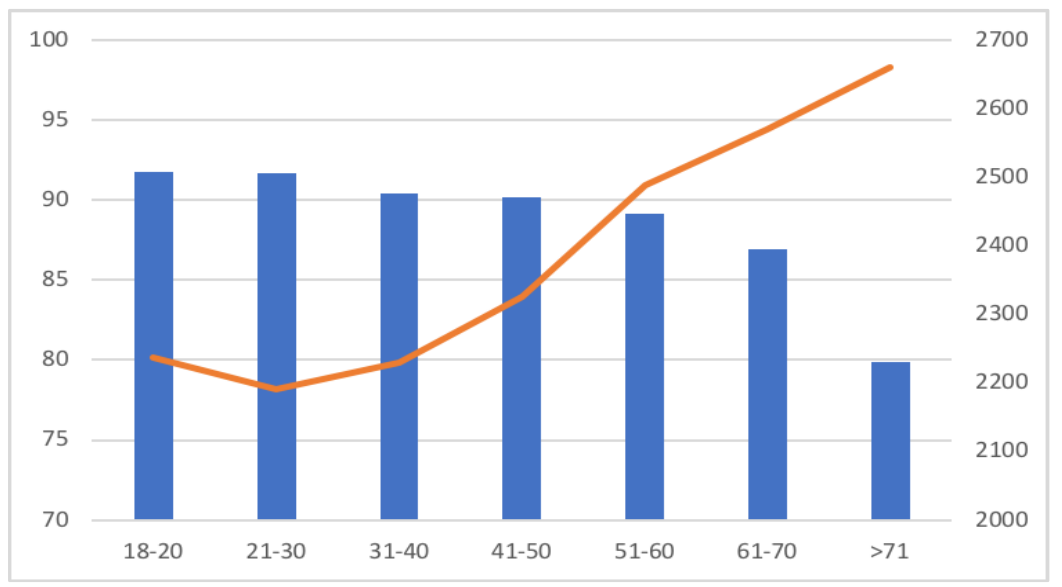

Fig. 2. PUT percent of correct reactions (blue bars) and detection tome (red line) in age groups.

Table 1. Relationship between age and PUT scores (oneway ANOVA results).

\begin{tabular}{llrrrrc}
\hline & & \multicolumn{1}{c}{ SS } & df & \multicolumn{1}{c}{ MS } & F & p \\
\hline Mean inspection & Between & 204399945,6 & 6 & 34066657,6 & 101,0 & $<, 001$ \\
time & Within & 5669503747,0 & 16799 & 337490,5 & & \\
& Total & 5873903692,0 & 16805 & & & \\
\hline Correct reactions & Between & 21537,8 & 6 & 3589,6 & 116,5 & $<, 001$ \\
$\%$ & Within & 517661,5 & 16800 & 30,8 & & \\
& Total & 539199,3 & 16806 & & & \\
\hline
\end{tabular}

The differences in processing speed and adequacy are robust (eta-square is 0,03 and 0,04 ). The Tukey's HSD test identified (in both variables) that subjects before 40 years of age are homogenous group, while older are divided into several sets. It shall be concluded that younger (less than 40) drivers are forming group of optimal performance. The 10. percentile in this group has been indicated as a cut point for attentional performance. Computed values are 1764 for inspection time and 90 for percent correct.

In the simulator experiment, age and attention accuracy were slightly correlated (Chisquare $=6,424, \mathrm{df}=2, \mathrm{p}=.040$ ), but only youngest group presented better performance, both in optimal and older drivers group percentage of subject with attention problems were similar and differences in those two groups were not significant.

Table 2. Crosstable age X Attention processing accuracy.

\begin{tabular}{lllll}
\hline & & \multicolumn{2}{l}{ Attention processing accuracy } & Total \\
\cline { 2 - 4 } & & Low & Accurate & \\
\hline \multirow{2}{*}{ Age group } & Less than 25 & 2 & 18 & 20 \\
& $30-45$ & 8 & 9 & 17 \\
\multirow{2}{*}{ Total } & Above 50 & 7 & 13 & 20 \\
& & 17 & 40 & 57 \\
\hline
\end{tabular}

According to above result, further analyses included attention accuracy as an independent variable.

The effect of experimental condition and attention accuracy were significant only in a few indicators. Results are presented in Table 3. 
Table 3. Influence of attention accuracy and exposition of quasi-advertisement stimuli on driving performance in $3 \mathrm{VPT}$ paradigm.

\begin{tabular}{|c|c|c|c|c|c|c|}
\hline Source & Variable & SS & $\mathrm{df}$ & $\mathrm{MS}$ & $\mathrm{F}$ & $\overline{\mathrm{p}}$ \\
\hline \multirow{8}{*}{$\begin{array}{l}\text { Exposition } \\
\text { time }\end{array}$} & mean speed & ,902 & 2 & ,451 & 3,458 &, 039 \\
\hline & speed SD & 3,1 & 2 & 1,554 & ,732 & ,486 \\
\hline & mean TTC & 28219543070000 & 2 & 14109771 & 1,323 & ,275 \\
\hline & & 0 & & 5400000 & & \\
\hline & TTC SD & 21245148690000 & 2 & 10622574 & ,913 & ,407 \\
\hline & & & & 350000 & & \\
\hline & $\begin{array}{l}\text { Mean distance to } \\
\text { centerline (DTC) }\end{array}$ & ,069 & 2 & ,034 & 1,244 & ,296 \\
\hline & DTC Std.dev. & ,002 & 2 & ,001 & ,148 &, 862 \\
\hline \multirow[t]{6}{*}{ Attention } & mean speed &, 086 & 1 & ,086 & ,661 &, 420 \\
\hline & speed SD & 12,420 & 1 & 12,420 & 5,853 & ,019 \\
\hline & mean TTC & 44252502010000 & 1 & $\begin{array}{r}44252502 \\
010000\end{array}$ & ,415 &, 522 \\
\hline & TTC SD & 17824055610000 & 1 & $\begin{array}{r}17824055 \\
610000\end{array}$ & 1,532 & ,221 \\
\hline & $\begin{array}{l}\text { Mean distance to } \\
\text { centerline (DTC) }\end{array}$ & 033 & 1 &, 033 & 1,191 & ,280 \\
\hline & DTC Std.dev. &, 003 & 1 &, 003 & ,389 &, 536 \\
\hline \multirow{6}{*}{$\begin{array}{l}\text { Exp. Time X } \\
\text { Attention } \\
\text { interaction }\end{array}$} & mean speed & ,220 & 2 & , 110 & 846 &, 435 \\
\hline & speed SD & 10,064 & 2 & 5,032 & 2,371 & ,103 \\
\hline & mean TTC & 69728342190000 & 2 & $\begin{array}{r}34864171 \\
090000\end{array}$ & ,327 &, 723 \\
\hline & TTC SD & 6742639375000 & 2 & $\begin{array}{r}33713196 \\
87000\end{array}$ & 290 & ,750 \\
\hline & $\begin{array}{l}\text { Mean distance to } \\
\text { centerline (DTC) }\end{array}$ &, 045 & 2 & ,023 & ,814 & ,448 \\
\hline & DTC Std.dev. & ,004 & 2 & ,002 & ,302 &, 741 \\
\hline
\end{tabular}

Mean speed was slightly lower, when a stimulus representing a roadside advertising was presented for two seconds. Attention does not moderate this effect. Subjects with poor attention performance, however, presented stronger speed variability. Other effects were not significant.

In LCT paradigm, the effect of exposition time and accuracy of attentional selection on driving performance was estimated in similar way as in 3VPT. Results are presented in table 4.

Table 4. Influence of attention accuracy and exposition of quasi-advertisement stimuli on driving performance in LCT paradigm.

\begin{tabular}{llrrrrr}
\hline Source & Variable & SS & df & MS & F & p \\
\hline Exposition time & Ref.range &, 472 & 2 &, 236 & 1,754 &, 184 \\
& Id. range &, 335 & 2 &, 167 & 1,562 &, 220 \\
& Reaction time &, 164 & 2 &, 082 &, 390 &, 680 \\
& Speed variab. &, 021 & 2 &, 010 &, 222 &, 802 \\
\hline Attention & Ref.range &, 118 & 1 &, 118 &, 878 &, 354 \\
& Id. range &, 005 & 1 &, 005 &, 047 &, 830 \\
& Reaction time &, 013 & 1 &, 013 &, 062 &, 805 \\
& Speed variab. &, 005 & 1 &, 005 &, 114 &, 737 \\
\hline
\end{tabular}




\begin{tabular}{lllllll} 
Exp.time X & Ref.range &, 023 & 2 &, 011 &, 084 &, 920 \\
Attention & Id. range &, 040 & 2 &, 020 &, 186 &, 831 \\
performance & Reaction time &, 233 & 2 &, 116 &, 553 &, 579 \\
& Speed variab. &, 019 & 2 &, 010 &, 204 &, 816 \\
\hline
\end{tabular}

The analysis revealed no significant influence of experimental variables on driving performance.

\section{Conclusion}

Effects observed in experiment were not as strong as expected. In LCT paradigm no effects were observed. In 3VPT paradigm speed control depends on exposition time of quasiadvertisement exposition, and speed variability is explained by accuracy in attention demanding task. In summary, one must conclude that exposition time of peripheral stimuli have no spectacular impact on driving performance.

Of course, lack of remarkable effect can be explained by simplicity of the experiment. In relatively simple and predictable road situation two seconds of distraction may be compensated. In more complex situations safety may be endangered, when a vehicle travels 50 meters during two-second distraction. One statistical comment must also be made: tested models assumed estimation of error variance in interaction cells, so the main effect (of quasiadvertisement) depends on context of attentional performance.

Second conclusion, although is not directly related to roadside advertising problem is also important for road safety. Results of the attention and age study give insight into problem of aging and road safety. The age-related differences are robust, and it shall be stressed, that problems with visual information processing can be remarkable even for people after 40 years of age. From the other side one must remark, that even such strong effect is not exceptionalness - many older people have perception and attentional resources better than many people in optimal age. Psychological assessment of drivers is therefore important both for younger and older, because in both groups real performance may be much different from what we expect it to be.

\section{References}

1. K. Young, M. Regan, Driver distraction: A review of the literature, in: I.J. Faulks, M. Regan, M. Stevenson, J. Brown, A. Porter, J.D. Irwin (Eds.), Distracted Driv., pp. 379-405 (NSW: Australasian College of Road Safety, Sydney, 2007)

2. D. Crundall, E. Van Loon, G. Underwood, Attraction and distraction of attention with roadside advertisements, Accid. Anal. Prev. 38 pp. 671-677 (2006). doi:10.1016/j.aap.2005.12.012

3. J. Fofanova, M. Vollrath, Distraction while driving: The case of older drivers, Transp. Res. Part F Traffic Psychol. Behav. 14 pp. 638-648 (2011). doi:10.1016/J.TRF.2011.08.005

4. D.J. Madden, Aging and Visual Attention, Curr. Dir. Psychol. Sci. 16 pp. 70-74 (2007). doi:10.1111/j.1467-8721.2007.00478.x

5. I. Kołodziejczyk, Neuropsychologia starzenia poznawczego, Kosmos. 56 pp. 49-62 (2007)

6. A. Mcmonagle, Traffic accidents and roadside features, Highw. Res. Board Bull. 55 pp. 38-48 (1950)

7. S. Bendak, K. Al-Saleh, The role of roadside advertising signs in distracting 
drivers, Int. J. Ind. Ergon. 40 pp. 233-236 (2010). doi:10.1016/j.ergon.2009.12.001

8. A. Olejniczak-Serowiec, N. Maliszewski, K. Ziętek, Social attitudes towards roadside advertising, MATEC Web Conf. 122 pp. 03006 (2017). doi:10.1051/matecconf/201712203006

9. B. Wallace, Driver distraction by advertising: genuine risk or urban myth?, Munic. Eng. 156 pp. $185-190$ (2003)

10. A. Treisman, Feature binding, attention and object perception, in: G.W. Humphreys, J. Duncan, A. Treisman (Eds.), Attention, Sp. Action, pp. 91-111 (Oxford University Press, Oxford, 1999)

11. A. Tarnowski, Test2Drive. Podręcznik użytkownika (Alta ltd., Siemianowice Śląskie, 2014)

12. K. Gąsiorek, A. Tarnowski, J. Harasimczuk, The influence of attention distraction on driver behavior, in this volume, (n.d.)

13. S. Mattes, The lane-change-task as a tool for driver distraction evaluation, Qual. Work Prod. Enterp. Futur. 57 (2003)

14. M. Kruszewski, M. Niezgoda, T. Kamiński, A. Matysiak, M. Nader, Pilot Study over Secondary Task Cognitive Workload Induced on Drivers in AS 1200-6 Simulator, Logist. Transp. 36 pp. 69-78 (2017) 